\section{Projections in Abstract Sets}

By

\section{E. Marczewski (Wrocław) and C. Ryll-Nardzewski (Warszawa)}

1. Problems and results. Let $X$ and $Y$ be two fixed abstract sets. For every subset $Z$ of the Cartesian product $X \times Y$ let us denote by $\mathrm{P}(Z)$ the projection of $Z$ on $\bar{X}$; in other terms $x \in \mathrm{P}(Z)$ if and only if there exists $y \in Y$ such that $(x, y) \in Z$.

The operation $\mathrm{P}$ is absolutely additive:

$$
\mathrm{P}\left(\sum_{t} Z_{t}\right)=\sum_{t} \mathrm{P}\left(Z_{t}\right)
$$

but it is not multiplicate. We have

$$
\mathrm{P}\left(\prod_{t} Z_{t}\right) \subset \prod_{t} \mathrm{P}\left(Z_{t}\right)
$$

while the converse inclusion is true only under some additional assumptions, e.g. in the fundamental lemma (Section 2) and in the following obvious proposition:

(3) If $A_{j} \subset X, B_{j} \subset Y$ and $B_{1} B_{2} \ldots \neq 0$, then $\mathrm{P}\left[\left(A_{1} \times B_{1}\right)\left(A_{2} \times B_{2}\right) \ldots\right]=A_{1} A_{2} \ldots$

For every class $Q$ of sets let us denote respectively

\begin{tabular}{c|c|c|c|c} 
by & $Q_{s}$ & $Q_{d}$ & $Q_{v}$ & $Q_{\delta}$ \\
\hline $\begin{array}{c}\text { the class of all } \\
\text { sets of the form }\end{array}$ & $Q_{1}+Q_{2}+\ldots+Q_{n}$ & $Q_{1} Q_{2} \ldots Q_{n}$ & $Q_{1}+Q_{2}+\ldots$ & $Q_{1} Q_{2} \ldots$
\end{tabular}

where $n=1,2, \ldots$ and $Q_{j} \in Q$.

Let $\boldsymbol{E}$ denote a class of subsets of $X, \boldsymbol{H}^{\mathrm{T}}$ a class of subsets of $Y$, and $\boldsymbol{H}$ the class of all sets $E \times F$, where $E \in \boldsymbol{E}$ and $\boldsymbol{F} \in \boldsymbol{F}$.

It follows easily from (1) and (3) that

\begin{tabular}{c|cccccccc} 
If $0 \neq Z_{\epsilon}$ & $\boldsymbol{H}$ & $\boldsymbol{H}_{s}$ & $\boldsymbol{H}_{d}$ & $\boldsymbol{H}_{\sigma}$ & $\boldsymbol{H}_{\dot{\delta}}$ & $\boldsymbol{H}_{s d}=\boldsymbol{H}_{d s}$ & $\boldsymbol{H}_{\delta s}$ & $\boldsymbol{H}_{d \sigma}$ \\
\hline then $\mathrm{P}(\boldsymbol{Z}) \epsilon$ & $\boldsymbol{E}$ & $\boldsymbol{E}_{s}$ & $\boldsymbol{E}_{d}$ & $\boldsymbol{E}_{\sigma}$ & $\boldsymbol{E}_{\delta}$ & $\boldsymbol{E}_{s d}=\boldsymbol{E}_{d s}$ & $\boldsymbol{E}_{\delta s}$ & $\boldsymbol{H}_{d \sigma}$
\end{tabular}

Thus the following problem arises: what can be said of the projection of a set $Z \in \boldsymbol{H}_{s \delta}$ ? The answer is simple: nothing in general. If $X=Y=$ the unit interval, if $\boldsymbol{E}$ is the class of all closed subintervals of $X$ and if $F$ is the class of all subsets of $Y$, then for ererr $A C X$ there is a set $C \in \boldsymbol{H}_{s \delta}$ such that $\mathrm{P}(C)=A$.

In fact, denoting by $D$ the diagonal, $i$. e. the set of points $(x, x)$, where $x \in X$, and setting

$$
\begin{gathered}
C(A \times A) \cdot D, \quad I_{m}^{n}=\frac{m-1}{n}, \frac{m}{n}, \quad \text { for } \quad m=1,2, \ldots, n ; \quad n=1,2, \ldots \\
\text { and } \begin{array}{c}
C_{n}=\left[I_{1}^{n} \times\left(A I_{1}^{n}\right)\right]+\left[I_{2}^{n} \times\left(A I_{2}^{n}\right)\right]+\ldots+\left[I_{n}^{n} \times\left(A I_{n}^{n}\right)\right]
\end{array}
\end{gathered}
$$

we obtain

$$
C_{n} \in \boldsymbol{H}_{s}, \quad C=C_{1} C_{2} \ldots \in \boldsymbol{H}_{s \delta}, \quad \mathrm{P}(C)=A .
$$

The purpose of this paper ${ }^{1}$ ) is to prove that, nevertheless, under some assumptions relating to $F$, the projections of sets belonging to $\boldsymbol{H}_{s \delta}, \boldsymbol{H}_{\sigma \delta}$, etc. belong to some classes determined by the class $\boldsymbol{E}$ only.

The notion which plays an essential role in what follows is that of a compact class of sets ${ }^{2}$ ). A class $\boldsymbol{F}$ is compact, if, for erery sequence $F_{n} \in \boldsymbol{F}$, we have $F_{1} F_{2} \ldots \neq 0$ whenerer $F_{1} F_{2} \ldots F_{n} \neq 0$ for $n=1,2, \ldots$ Consequently a multiplicative class $F$ (i.e. such that $\boldsymbol{F}_{d}=\boldsymbol{F}$ ) is compact if and only if every descending sequence of non-roid sets $F_{n} \in \boldsymbol{F}$ has a nonvoid product.

Next, denote by $Q_{A}$ the class of all sets of the form $\Sigma Q_{k_{1}} Q_{k_{1} k_{2} \ldots} \ldots$ where $Q_{k_{1} k_{2} \ldots k_{n}} \in Q$ and the summation extends over all infinite sequence $k_{1}, k_{2}, \ldots$ of natural numbers. It is well known that this operation, termed operation (A), has the following properties ${ }^{3}$ ):

$$
Q_{A} \supset Q_{\sigma}+Q_{\delta}, \quad Q_{A A}=Q_{A} .
$$

The result of this paper is

Theorem. If the class $\boldsymbol{F}$ is compact, then

\begin{tabular}{c|c|c|c} 
& $\mathrm{I}$ & $\mathrm{II}^{\prime}$ & $\mathrm{UI}$ \\
\hline if $0 \neq Z \epsilon$ & $\boldsymbol{H}_{s \dot{\delta}}$ & $\boldsymbol{H}_{\sigma \delta}$ & $\boldsymbol{H}_{A}$ \\
\hline then $\mathrm{P}(Z) \epsilon$ & $\boldsymbol{E}_{s \delta}$ & $\boldsymbol{E}_{A}$ & $\boldsymbol{E}_{A}$
\end{tabular}

1) Presented to the Polish Mathematical Society, Wroclaw Section, on April 1, 1952.

2) Cf. E. Marczewski, On compact measures, Fundamenta Mathematicae 4 (1953), p. 113-124, especially p. 115.

3) Cf. F. Hausdorff, Mengenlehre, Berlin-Leipzig 1935, p. 90-93.

Fundamenta Mathematicae T. XL. 
Notice that the implication $\mathrm{II}^{\prime}$ (which is an easy consequence of II and (4)) cannot be strengthened. Indeed, in case $\boldsymbol{E}=\boldsymbol{F}=$ the class of all closed subintervals of the unit interval $I$, the projections of all sets belonging to $\boldsymbol{H}_{\sigma s}$ constitute the class of all analytical subsets of $I$, or, in other words, the whole class $\boldsymbol{E}_{A}$.

The theorem will be applied in a forthcoming paper on measures in product spaces.

2. Fundamental lemma. For $Z \subset X \times Y$ and $x \in X$ denote by $\mathrm{S}_{x}(Z)$ the vertical section of $Z$ corresponding to $x$; in other terms, $y \in \mathrm{S}_{x}(Z)$ if and only if $(x, y) \in Z$. We shall prove that

If $Z_{1} \supset Z_{2} \supset \ldots$, where $Z_{j} \subset X \times Y$ for $j=1,2, \ldots$, and if for every $x \in X$ the sequence $S_{x}\left(Z_{j}\right)$ forms a compact class, then $\mathrm{P}\left(Z_{1} Z_{2} \ldots\right)=\mathrm{P}\left(Z_{1}\right) \cdot \mathrm{P}\left(Z_{2}\right) \cdot \ldots$

In virtue of $(2)$, it suffices to prove that

$$
\mathrm{P}\left(Z_{1} Z_{2} \ldots\right) \supset \mathrm{P}\left(Z_{1}\right) \cdot \mathrm{P}\left(Z_{2}\right) \cdot \ldots
$$

Let $x \in \mathrm{P}\left(Z_{1}\right) \cdot \mathrm{P}\left(Z_{2}\right) \cdot \ldots$ Consequently the sets

$$
\mathbf{S}_{x}\left(Z_{1}\right) \supset \mathbf{S}_{x}\left(Z_{2}\right) \supset \ldots
$$

arenon-void and, since they form a compact class, there is an $y$ such that

$$
y \in \mathbb{S}_{x}\left(Z_{1}\right) \cdot \mathbb{S}_{x}\left(Z_{2}\right) \cdot \ldots
$$

or, in other words, $(x, y) \in Z_{1} Z_{2} \ldots$ whence $x \in \mathrm{P}\left(Z_{1} Z_{2} \ldots\right)$, q. e. d.

3. The operation (A). For any system of sets $3=\left\{Z_{k_{1} k_{2} \ldots k_{n}}\right\}$ where $\left(k_{1}, k_{2}, \ldots, k_{n}\right)$ runs over the set of all finite sequences of natural numbers let us set

$$
\mathbf{A}(3)=\sum_{\left\{k_{j}\right\}} Z_{k_{1}} Z_{k_{1} k_{2}} \ldots
$$

where the summation extends over all infinite sequences $\left\{k_{j}\right\}$.

For every class $Q$ of sets, $Q_{A}$ is by definition the class of all sets $\mathbf{A}(3)$, where 3 runs over all systems of sets belonging to $\boldsymbol{Q}$.

A system $\left\{Z_{k_{1} k_{2} \ldots k_{n}}\right\}$ of sets will be called monotone, if we always have

$$
Z_{k_{1} k_{2} \ldots k_{n} k_{n+1}} \subset Z_{k_{1} k_{2} \ldots k_{n}}
$$

The following lemma is essential for projection properties:

If $0 \neq Z \in \boldsymbol{Q}_{A}$, then there exists a monotone system $3=\left\{Z_{k_{1} k_{2} \ldots k_{n}}\right\}$ of non-void sets belonging to $Q_{d}$ such that $Z=\mathrm{A}(3)$.

I shall outline the proof of this lemma.

Since $Z \in Q_{A}$, there is a system $\mathfrak{U}=\left\{A_{k_{1} k_{2} \ldots k_{n}}\right\}$. of sets belonging to $Q$ such that $Z=\mathrm{A}(\mathfrak{I})$.

Let us set

$$
B_{k_{1} k_{2} \ldots k_{n}}=A_{k_{1}} A_{k_{1} k_{2} \ldots A_{k_{1} k_{2} \ldots k_{n}}}
$$

obviously $\mathfrak{B}=\left\{B_{k_{1} k_{2} \ldots k_{n}}\right\}$ is a monotone system of sets belonging to $\boldsymbol{Q}_{d}$ and we have $Z=A(\mathfrak{B})$.

Next, denote by $M$ the set of all infinite sequences $\left\{k_{j}\right\}$ of natural numbers such that

Hence

$$
B_{k_{1}} B_{k_{1} k_{2}} B_{k_{1} k_{2} k_{3}} \ldots \neq 0
$$

$$
Z=\sum_{\left\{k_{j}\right\} \in M} B_{k_{1}} B_{k_{1} k_{2}} \ldots
$$

where the summation extends only over sequences $\left\{k_{j}\right\}$ belonging to $M$.

By a suitable new numeration of the sets, $B_{k_{1} k_{2} . . k_{n}}$ appearing in this sum, namely by repeating some sets satisfying (5), we oltain the required system 3 .

4. Proof of Theorem. I. Suppose $0 \neq Z \in \boldsymbol{H}_{s \sigma}$. Thus we may write $Z=Z_{1} Z_{2} \ldots$, where $Z_{1} \supset Z_{2} \supset \ldots$ and $Z_{j} \in \boldsymbol{H}_{d s}$, whence for every $x \in X$ we have

$$
\mathrm{S}_{x}\left(Z_{j}\right) \in F_{d s} \quad \text { or } \quad \mathrm{S}_{x}\left(Z_{j}\right)=0 .
$$

Since $\boldsymbol{F}$ is compact by hypothesis, the class $\boldsymbol{F}_{d s}$ is also compact ${ }^{4}$ ), and by the fundamental lemma

$$
\mathrm{P}(Z)=\mathrm{P}\left(Z_{1}\right) \cdot \mathrm{P}\left(Z_{2}\right) \cdot \ldots \in \boldsymbol{E}_{d s \delta}=\boldsymbol{E}_{s \delta}, \quad \text { q. e. d. }
$$

II. Suppose $0 \neq \boldsymbol{Z} \in \boldsymbol{H}_{A}$. BY lemma 3 there is a system 3 of nonvoid sets $\left.{ }^{5}\right)$

such that

$$
Z_{k_{1} k_{2} \ldots k_{n}}=E_{k_{1} k_{2} \ldots k_{n}} \times F_{k_{1} k_{2} \ldots k_{n}}
$$

$$
\begin{gathered}
E_{k_{1} k_{2} \ldots k_{n}} \in \boldsymbol{E}_{d}, \quad F_{k_{1} k_{2} \ldots k_{n}} \in \boldsymbol{F}_{d}, \\
E_{k_{1} k_{2} \ldots k_{n} k_{n+1}} \subset E_{k_{1} k_{2} \ldots k_{n}}, \quad F_{k_{1} k_{2} \ldots k_{n} k_{n+1}} \subset F_{k_{1} k_{2} \ldots k_{n}}, \\
Z=\mathrm{A}(3) .
\end{gathered}
$$

Since, for every sequence $\left(k_{1}, k_{2}, \ldots, k_{n}\right)$ we have

$$
\mathrm{S}_{x}\left(Z_{k_{1} k_{2} \ldots k_{n}}\right)=F_{k_{1} k_{2} \ldots k_{n}} \quad \text { or } \quad \mathrm{S}_{x}\left(Z_{k_{1} k_{2} \ldots k_{n}}\right)=0,
$$

$\left.{ }^{4}\right)$ (Cf. E. Marczewski, 1. c. ${ }^{2}$ ), theorems 2 (ii) and 2 (iii).

s) The second part of the lemma (i.e. the statement that $Z_{k_{1} k_{2} \ldots k_{n}}$ are non-void) is superflous in the case when the empty set belongs to $E$. Then it suffices to consider a monotone system $B$, such that the sets $E_{k_{1} k_{2} \ldots k_{n}}$ and $F_{k_{1} k_{2} \ldots k_{n}}$ are both roid or both non-roid. 
for every $x \in X$ all the sections $S_{x}\left(Z_{k_{1} k_{2} \ldots k_{n}}\right)$ form a compact class. Thus, for erery infinite sequence $\left(k_{1}, k_{2}, \ldots\right)$, we may apply the fundamental lemma to the sequence of sets $Z_{k_{1}} \supset Z_{k_{1} k_{2}} \supset \ldots$ Since $\mathrm{P}(A \times B)=A$, whenever $B$ is non-roid, we obtain

$$
\mathrm{P}(Z)=\sum_{i k_{j} ;} \mathrm{P}\left(\boldsymbol{Z}_{k_{1}} Z_{k_{1} k_{2}} \ldots\right)=\sum_{i k_{j} j} E_{k_{1}} E_{k_{1} k_{2}} \ldots \in \boldsymbol{H}_{d / A}=\boldsymbol{E}_{A}, \quad \text { q. e. d. }
$$

Note. The assumption in II of the compactness of $\boldsymbol{F}$ may be replaced by the following: there is a compact class $\boldsymbol{F}^{*}$ such that $\boldsymbol{F} \dot{C} \boldsymbol{F}_{A}^{*}$. In fact, if $\boldsymbol{H}^{*}$ denotes the class of all sets $E \times F$ where $E \in \boldsymbol{E}$ and $F \in F^{*}$, then it is easily seen that $\boldsymbol{H}_{A}^{*} \supset \boldsymbol{H}$, whence, by (4), $\boldsymbol{H}_{A}^{*}=\boldsymbol{H}_{A}$. Thus we reduce the generalized form of II from the original one, applied to the classes $\boldsymbol{E}, \boldsymbol{F}^{*}$ and $\boldsymbol{H}^{*}$.

Instytut Matematyezny Universytetu Wrocławskiego Mathematical Institute of the Wroclaw University

Remarks on the Compactness and non Direct Products
of Measures ${ }^{*}$ ) By

E. Marczewski (Wroclaw) and C. Ryll-Nardzewski (Warszawa)

Introduction. The direct product of two normalized measures $u$ in $X$ and $v$ in $X, i$.e. the measure $\lambda$ in $X \times Y$ such that

$$
\hat{\lambda}(A \times B)=\mu(A) \cdot v(B),
$$

has many regularity properties, e. $g$.

I. If $\mu$ and $v$ are countably additive, so is $\lambda^{1}$ ).

II. If $\mu, v$ and $\lambda$ are $\sigma$-measures, then

$$
\lambda_{i}(E \times Y)=u_{i}(E) \text { for } E \subset x
$$

(where, for every measure $\mu$, the symbol $\mu_{i}$ denotes the inner measure induced by $\mu)^{2}$ ).

In sections 1 and 2 of this paper we deal with the same propositions for non-direct products, i.e. when the condition (1) is replaced by the weaker ones:

$$
\lambda(A \times Y)=\mu(A), \quad \lambda(X \times B)=\nu(B) .
$$

It turns out that the propositions $\mathrm{I}$ and $I I$ for the nondirect products remain true under the additional assumption that the measure $v$ is compact, and that they are false in general.

Sections 3 and 4 are further contributions to the study of compact measures. It is known that the minimal $\sigma$-extension of a compact measure is also compact. Here we show that the converse of this theorem is not true.

We apply theorems on compact measures proved in the paper: Marczewski [3] (quoted below as $\mathbf{C}$ ), and theorems on projections proved in the paper: Marczewski and Ryll-Nardzewski [4] (quoted as $\mathrm{P}$ ).

*) Presented to the Polish Mathematical Society, Wrockaw Sect., on April 1, 1952. 1) This follows $e . g$. from the existence of the direct $\sigma$-product of any two $\sigma$-measures, see e.g. Halmos [2], p. 144, Theorem B.

2) This follows e. g. from the abstract Fubini theorem. See ibidem. 